\title{
Impact of Velvet Complex on Transcriptome and Penicillin G Production in Glucose-Limited Chemostat Cultures of a $\beta$-Lactam High-Producing Penicillium chrysogenum Strain
}

\author{
Tânia Veiga,,2 Jeroen G. Nijland, ${ }^{2,3}$ Arnold J.M. Driessen, ${ }^{2,3}$ Roel A.L. Bovenberg,,3,4 \\ Hesselein Touw, ${ }^{4}$ Marco A. van den Berg, ${ }^{4}$ Jack T. Pronk,${ }^{1,2}$ and Jean-Marc Daran ${ }^{1,2}$
}

\begin{abstract}
The multicomponent global regulator Velvet complex has been identified as a key regulator of secondary metabolite production in Aspergillus and Penicillium species. Previous work indicated a massive impact of PcvelA and PclaeA deletions on penicillin production in prolonged batch cultures of P. chrysogenum, as well as substantial changes in transcriptome. The present study investigated the impact of these mutations on product formation and genome-wide transcript profiles under glucose-limited aerobic conditions, relevant for industrial production of $\beta$-lactams. Predicted amino acid sequences of PcVelA and PcLaeA in this strain were identical to those in its ancestor Wisconsin54-1255. Controls were performed to rule out transformation-associated loss of penicillin-biosynthesis clusters. The correct PcvelA and PclaeA deletion strains revealed a small reduction of penicillin $G$ productivity relative to the reference strain, which is a much smaller reduction than previously reported for prolonged batch cultures of similar P. chrysogenum mutants. Chemostat-based transcriptome analysis yielded only 23 genes with a consistent differential response in the PcvelA $\Delta$ and PclaeA $\Delta$ mutants when grown in the absence of the penicillin $\mathrm{G}$ side-chain precursor phenylacetic acid. Eleven of these genes belonged to two small gene clusters, one of which contained a gene with high homology to the aristolochene synthase. These results provide a clear caveat that the impact of the Velvet complex on secondary metabolism in filamentous fungi is strongly context dependent.
\end{abstract}

\section{Background}

A SCOMYCETOUS FILAMENTOUs fungi produce a large range of structurally diverse, complex low-molecular-weight compounds known as secondary metabolites. Secondary metabolites are generally nonessential for survival and highly species or even strain specific (Keller et al., 2005) but may positively affect growth, physiology, or reproduction of the producing organism (Demain and Fang, 2000; Fox and Howlett, 2008; Luckner, 1991; von Dohren, 2004). In addition, several fungal secondary metabolites are of applied interest, for example, because of their role as toxins or their application as antibiotics (Demain and Adrio, 2008; Manzoni and Rollini, 2002; van der Beek and Roels, 1984).

The genes involved in fungal secondary metabolic pathways share a tendency towards physical clustering, with a preference for subtelomeric regions (Galagan et al., 2005; van den Berg et al., 2008). Evidence is mounting that this spatial organization contributes to regulation of these pathways. Subtelomeric gene regulation was observed in Aspergillus parasiticus, in which chromosomal translocation of a secondary metabolite gene cluster resulted in deregulated expression (Chiou et al., 2002). Expression of the A. parasiticus ver-1 and nor-1 genes, involved in aflatoxin biosynthesis, was shown to depend strongly on chromosomal localization, thereby revealing a cellular strategy for activation or repression of entire biosynthetic gene clusters (Palmer and Keller, 2010; Shwab and Keller, 2008). Over the past years, several reports established epigenetic regulation of cryptic secondary metabolite clusters, in which coexpression of the clustered genes was directly affected by chromosomal remodeling. In $A$. nidulans, deletion of $c c l A$, a $B R E 2$ ortholog involved in histone $\mathrm{H} 3$

${ }^{1}$ Department of Biotechnology, Delft University of Technology, Delft, The Netherlands.

${ }^{2}$ Kluyver Centre for Genomics of Industrial Fermentation, Delft, The Netherlands.

${ }^{3}$ Department of Molecular Microbiology, University of Groningen, AG Groningen, The Netherlands.

${ }^{4}$ DSM Biotechnology Center, Delft, The Netherlands. 
lysine 4 methylation, resulted in activation of the expression of secondary metabolite clusters (Bok et al., 2009).

The Velvet complex, an intensively studied multicomponent global regulator, is involved in morphogenesis as well as in the regulation of a wide range of secondary metabolite pathways in filamentous fungi (Bayram et al., 2008; Kosalkova et al., 2009). In A. nidulans, the core of the complex is composed of three subunits VeA, VelB, and LaeA (Bayram et al., 2008). The Velvet protein VeA has been identified as a positive transcriptional regulator of genes involved in secondary metabolism and as a negative regulator of asexual development (Dreyer et al., 2007; Kato et al., 2003; Krappmann et al., 2005). LaeA is a methyltransferase involved in activation of secondary metabolism genes through epigenetic control (Bayram et al., 2008; Keller et al., 2005; ReyesDominguez et al., 2010). VelB, a VeA-like protein, seems to be necessary for recruiting the regulator of sporogenesis VosA. Through VeA, the complex interacts with light responsive proteins, FhpA, LreA, and LreB or the $\alpha$-importin KapA (Bayram et al., 2008).

Orthologs of the Velvet complex components in several fungi show a conservation of amino acid sequence and function, but also some degree of flexibility (Calvo, 2008; Hoff et al., 2010). As previously reported (Dreyer et al., 2007), in $P$. chrysogenum the predicted protein sequence encoded by the ORF Pc13g13200 exhibited 61\% sequence similarity and $48 \%$ sequence identity with $A$. nidulans VelA. The predicted protein sequence of Pc16g14010 showed a 55\% sequence identity to the amino acid sequence of $A$. nidulans LaeA. In P. chrysogenum, PclaeA (Pc16g14010), and PcvelA (Pc13g13200) knockdown mutants have been constructed in two strain backgrounds with different penicillin biosynthesis ability: Wisconsin54-1255 (an early strain in the improvement program) and P2niaD (a penicillin high-producing strain derived from the Panlabs strain improvement program) (Newbert et al., 1997). The PclaeA deletion resulted in transcriptional downregulation of all genes involved in penicillin biosynthesis and a drastic reduction of penicillin production (by circa $80 \%$ ) (Hoff et al., 2010; Kosalkova et al., 2009). Deletion of PcvelA in the high-producing strain had a similar impact (Hoff et al., 2010). Moreover, transcriptome analysis of PclaeA and PcvelA deletions in strain P2niaD revealed a widespread impact, with over $10 \%$ of the genome being affected by these deletions, with a clear overrepresentation of genes involved in secondary metabolite pathways and in fungal development (Hoff et al., 2010). These observations are in line with studies on other filamentous fungi, in which deletion of veA or laeA was similarly shown to lead to downregulation of the production of several secondary metabolites (Calvo, 2008; Dreyer et al., 2007; Horwood et al., 2004; Wiemann et al., 2010).

Previous studies on the impact of the Velvet complex in $P$. chrysogenum were performed in prolonged batch cultures. Time course analysis revealed that the impact of PcvelA and PclaeA mutations was most pronounced after prolonged incubation (Hoff et al., 2010), but the physiological status of these cultures was not precisely defined. Industrial production of $\beta$-lactam antibiotics is performed in sugar-limited, aerobic fed-batch cultures (Menezes et al., 1994). The aim of the present study is to characterize the genetic basis of the Velvet complex in the $P$. chrysogenun high penicillin producing strain DS17690 and to investigate its impact on physiology, penicillin production, and transcriptional regulation under industrially relevant conditions. To this end, we studied the impact of PcvelA and PclaeA deletions in aerobic, glucose-limited chemostat cultures of the penicillin highproducing strain P. chrysogenum DS17690.

\section{Methods}

\section{Strains}

Penicillium chrysogenum strains used in this study are listed in Table 1. DS17690 is a high penicillin producing strain, resulting from the DSM classical strain improvement program (DSM Anti-Infectives, Delft, The Netherlands). P. chrysogenum

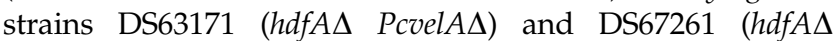
$P c l a e A \Delta$ ) were derived from strain DS54465 (hdfAD) (Snoek et al., 2009), which has a high frequency of homologous recombination. Requests for academic use of the P. chrysogenum strains, under a material transfer agreement, should be addressed to Prof. R.A.L. Bovenberg (DSM Anti-Infectives, P.O. Box 425, Delft NL-2600 AK, The Netherlands).

\section{Deletion cassette construction}

All $P$. chrysogenum gene fragments were amplified from genomic DS17690 DNA using Phusion Hot-Start polymerase (Finnzymes, Landsmeer, The Netherlands). Vector pDONR ${ }^{\mathrm{TM}}$ P4-P1R (Invitrogen, Breda, The Netherlands) was used to clone the 782-bp 5'-flanking region of the Pc16g14010 (PclaeA) gene. This fragment was PCR amplified with primers attB4F5 Pc16g14010HindIII and attB1R4 Pc16g14010 (Table 2). The vector and the fragment were recombined with $\mathrm{BP}$ clonases (Invitrogen) yielding pDONR41-5'FR 16g14010. pDONR ${ }^{\mathrm{TM}}$ P2-P3R (Invitrogen) was used to clone the 981-bp 3'-flanking region of the Pc16g14010 gene. This fragment was PCR amplified with the primer pair attB2F Pc16g14010/attB3R Pc16g14010 HindIII. The vector and the fragment were recombined with BP clonase yielding pDONR23-3'FR 16g14010. pDONR $^{\mathrm{TM}} 221$ Gateway $^{\circledR}{ }^{\circledR}$ vector (Invitrogen) was used to clone the $A$. nidulans acetamidase-gene (amdS) gene under control of the A. nidulans gpdA promoter and penDE terminator, which were amplified from plasmid pBlueAMDS (Nijland et al., 2010) with primers attB1F AMDS and attB2R AMDS. The three fragments were then combined with the $\mathrm{pDEST}^{\mathrm{TM}} \mathrm{P} 4-\mathrm{R} 3$ in the LR reaction of the Multisite Gateway ${ }^{\circledR}$ Three-Fragment Vector Construction Kit (Invitrogen)

Table 1. P. chrysogenum Strains Used in This Study

\begin{tabular}{|c|c|c|}
\hline Strain & $\begin{array}{l}\text { Genotype/ } \\
\text { description }\end{array}$ & Reference \\
\hline DS17690 & $\begin{array}{l}\text { High penicillin } \\
\text { producer }\end{array}$ & $\begin{array}{l}\text { (Harris et al., } \\
\text { 2007) }\end{array}$ \\
\hline $\begin{array}{l}\text { Wisconsin } \\
\text { 54-1255/ } \\
\text { ATCC28089 }\end{array}$ & $\begin{array}{l}\text { Ancestor of } \\
\text { DS17690 }\end{array}$ & $\begin{array}{l}\text { (Macdonald } \\
\text { et al., 1964) }\end{array}$ \\
\hline DS54465 & $h d f A \Delta$ & $\begin{array}{l}\text { (Snoek I.S. } \\
\text { et al., 2009) }\end{array}$ \\
\hline DS50652 & $\begin{array}{l}{[p c b A B-} \\
\quad p c b C-p e n D E] \Delta\end{array}$ & $\begin{array}{l}\text { (Nijland } \\
\text { et al., 2010) }\end{array}$ \\
\hline DS63171 & 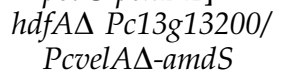 & This work \\
\hline DS67261 & $\begin{array}{l}\text { hdfA } P \text { Pc16g14010/ } \\
\text { PclaeAs-amdS }\end{array}$ & This work \\
\hline
\end{tabular}


Table 2. Primers Used in This Study

\begin{tabular}{|c|c|}
\hline Primer & Sequence $\left(5^{\prime}-3^{\prime}\right)$ \\
\hline attB4F5 Pc16g14010HindIII & GGGGACAACTTTGTATAGAAAAGTTGAAGCTTCAACCTCTAGTTACCGGTAGCGCG \\
\hline attB1R4 Pc16g14010 & GGGGACTGCTTTTTTGTACAAACTTGGCGTTCGAGGCGTGGGATGCCTG \\
\hline attB2F Pc16g14010 & GGGGACAGCTTTCTTGTACAAAGTGGGTGAAGCATAGCAATCGACCGCC \\
\hline attB3R Pc16g14010 HindIII & GGGGACAACTTTGTATAATAAAGTTGGTTGGTCTACAATCCGGCGTTGGG \\
\hline attB1F AMDS & GGGGACAAGTTTGTACAAAAAAGCAGGCTCGCAGGAATTCGAGCTCTGTAC \\
\hline attB2R AMDS & GGGGACCACTTTGTACAAGAAAGCTGGGTCTCGCTCGTACCATGGGTTGAG \\
\hline F-amds & GAAAGTCCAGACGCTGCCTGCG \\
\hline R-amds & CCCTGGTGGCATATGTTAGCTG \\
\hline F14010 & GTGCTATGGCTAACTGGTACTCG \\
\hline R14010 & TTTCGCGCTTGATAGATGTGCAG \\
\hline F $\gamma$-actin gDNA & TTCTTGGCCTCGAGTCTGGCGG \\
\hline $\mathrm{R} \gamma$-actin gDNA & GTGATCTCCTTCTGCATACGGTCG \\
\hline F-IGR Pc20g07090 & GTTCCTATAGGACGTAGCTCCGC \\
\hline R-IGR Pc20g07090 & AAATCAGCTCTACTAGCGATCCGC \\
\hline F-pcbAB & CACTTGACGTTGCGCACCGGTC \\
\hline R-pcbAB & CTGGTGGGTGAGAACCTGACAG \\
\hline F-pcbC & AGGGTTACCTCGATATCGAGGCG \\
\hline R-pcbC & GTCGCCGTACGAGATTGGCCG \\
\hline PVF & CCTTCGCCGACTGAGGAGTACGGAGTAGCTTGCGGTGACTTTCATTC \\
\hline PVR & TCTTAGACGCTCCGGAGCATAGAAGTTTCGCGGATTGATGTTGTTATTCCCAGATATTC \\
\hline OVF & ATGGCCAACAGACCATCTCTCATGCCACCTC \\
\hline OVR & CCTTCGCCGACTGATGTACTCGACGGAGGAGCTTTCATGACTATTTTG \\
\hline A3F & TTTTTGTCAAGCTTACATATGCCACCAGGGCTAC \\
\hline $\mathrm{A} 3 \mathrm{R}$ & $\begin{array}{l}\text { ATGGATGGATCCATAACTTCGTATAATGTATGCTATACGAAGTTATGT } \\
\text { TGAGTGGTATGGGGCCATCC }\end{array}$ \\
\hline A5F & $\begin{array}{l}\text { CTGGAATTGTTTAAACGCGGCCGCCGCCTGCAGGATAACTTCGTATAGCATAC } \\
\text { ATTATACGAAGTTATGACTCTTTCTGGCATGCGGAGAGAC }\end{array}$ \\
\hline A5R & TTCTGGAGAAGCTTGTCCCAGAGCTCGTTCATGTTAACAG \\
\hline TCF & CGAGGAGCACCTGCAGGCCGACGCCGACCAACACCGCC \\
\hline TCR & $\begin{array}{l}\text { CCGCCAGTGTTTAAACTAGCGGCCGCATGGCGCGCCGTATTGGGTGTTACGGAGCA } \\
\text { TTCAC }\end{array}$ \\
\hline TWF & TGCAGCGCGTTAGAATAC \\
\hline TWR & CGGATCCTTCGCCGACTGA \\
\hline F13g13200 & CAGTACCGAGTCCATGTATGCCGGG \\
\hline R13g13200 & GGGGGAAGTTTGTTGTGGTACCTGG \\
\hline RTamdS & GCCACAGGTGACTCTGGATGG \\
\hline FpgdA & GTCTCTCCGCATGCCAGAAAG \\
\hline veA $\mathrm{Fw}$ & CACCATGGCCAACAGACCATCTCTCATGC \\
\hline$v e A \operatorname{Rv}$ & TTATGTACTCGACGGAGGAGCTTTC \\
\hline LaeA Fw & CACCATGTCTTACCGAGAGTCATCTGGTTCCTTTC \\
\hline LaeA Rv & TTATTCCTCGACTGGTTTTCGCGCTTG \\
\hline
\end{tabular}

resulting in the destination vector (pDest43-KO 16g14010). This vector contains flanking HindIII restriction sites that were used to cut out the deletion cassette. The Pc16g14010/ PclaeA deletion cassette was transformed in DS54465 ( $h d f A \Delta$ ) protoplasts using the acetamidase gene ( $a m d S)$ as selection

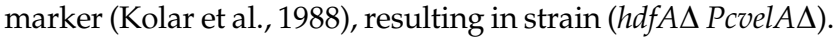

For the mutation of the Pc13g13200 (PcvelA) gene, the promoter and open reading frame (ORF) were PCR amplified using the primer pairs PVF/PVR and OVF/OVR, respectively
(Table 2). Both fragments contained a 14-bp tail to facilitate directional and efficient cloning, via the STABY method (Eurogentec, Maastricht, The Netherlands). Two variants of the standard STABY vector, pSTC1.3, were constructed for individual cloning of both fragments. pSTamdSL was constructed by insertion of a $1.8-\mathrm{kb}$ fragment of the amdS expression cassette in pSTC1.3. The fragment of $1.8 \mathrm{~kb}$ that contains the last $2 / 3$ of amdS was PCR amplified from the plasmid pHELY-A1 (van den Berg et al., 2004) using primers 
A3F and A3R (Table 2). The fragment and the plasmid pSTC1.3 were then digested with the restriction enzymes HindIII-Bam $\mathrm{HI}$ and the two fragments were ligated overnight using T4 ligase (Invitrogen) as recommended by the STABY-protocol (Eurogentec) yielding the plasmid pSTamdSL. Similarly, the plasmid pSTamdSR was constructed by inserting an overlapping $2.4-\mathrm{kb}$ fragment covering the $A$. nidulans gpdA promoter and the first $2 / 3$ of the amdS expression cassette. The fragment was PCR amplified using pHELY-A1 as template and the primers A5F and A5R. In the process the EcoRV site found in the PgpdA sequence was removed. Subsequently, the PCR fragment and pSTC1.3 were digested with HindIII-PmeI. The two fragments were ligated as previously mentioned. Next, a strong terminator was inserted in front of the PgpdA-amdS. To do so, the 0.7-kb trpC terminator was PCR amplified using primers TCF and TCR (Table 2) from pAN7-1 (Punt et al., 1987) and introduced via the Sbfl-NotI sites of PgpdAamdS yielding the plasmid pSTamdSR. Both vectors contained an overlapping but nonfunctional fragment of the fungal selection marker amdS gene, encoding acetamidase and allowing recipient cells that recombine the two fragments into a functional selection marker to grow on agar media with acetamide as the sole nitrogen source (Tilburn et al., 1983). Plasmid pSTamdSL and pSTamdSR were transformed to chemically competent CYS21 Escherichia coli cells (Eurogentec). Transformants were used to PCR amplify the gene specific sequences fused to the nonfunctional amdS fragments using oligonucleotides TWF and TWR. The obtained PCR fragments were combined and used to transform P. chrysogenum DS54465 ( $h d f A \Delta$ ) (Snoek et al., 2009) protoplasts resulting in strain DS67261 (hdfA $\Delta$ PclaeAs).

\section{Confirmation of the mutations by Southern blotting}

Genomic DNA of four transformants and the DS54465 were isolated using the E.Z.N.A. Fungal DNA kit (Omega Biotek, Amsterdam, The Netherlands). Genomic DNA $(2.5 \mu \mathrm{g})$ was digested using the NdeI restriction enzyme, separated on a $0.8 \%$ agarose gel, and blotted onto a Zeta-Probe membrane (BioRad, Hercules, CA) (Sambrook et al., 1989). The 5'flanking region of the Pc16g14010 gene was used as probe, which was labeled with digoxigenin using the PCR DIG Probe Synthesis Kit (Roche, Paris, France) according to the manufacturer's instructions. Hybridization was done overnight at $42^{\circ} \mathrm{C}$ in hybridization buffer $(50 \%$ formamide, $5 \times$ SSC, $2 \%$ blocking reagent (Roche), 0.1\% Na-lauroylsarcosin, $0.02 \%$ SDS) and the membranes were washed twice with $2 \times$ SSC, $0.1 \%$ SDS for $15 \mathrm{~min}$, and twice with $0.2 \times$ SSC, $0.1 \%$ SDS. Digoxigenin-labeled probes were detected by chemiluminescent using CPD-star (Roche).

\section{Confirmation of the mutations by PCR}

To confirm the correct modification of the gene Pc13g13200 (PcvelA) with the use of a bipartite vector, the primer pairs and F13g13200-RtamdS, F13g13200-R13g13200, and FgpdAR13g13200 (Table 2) were used. To confirm the deletion in the strain PclaeAs, both the native Pc16g14010 gene and the Pc16g14010::amdS deletion were amplified using primers F14010 and R14010 (Table 2 and Figure 1).

\section{Penicillin gene cluster copy number determination by quantitative PCR analysis}

To analyze the number of penicillin biosynthetic gene clusters in the transformants (hdfAL PcvelA $\Delta$ ) and DS67261 ( $h d f A \Delta$ Pclae $A \Delta$ ), $\gamma$-actin (with the primers $\mathrm{F} \gamma$-actin gDNA and $\mathrm{R} \gamma$-actin gDNA) (Table 2) and an intergenic target (using the primers F-IGR Pc20g07090 and R-IGR Pc20g07090) (Table 2) were used as reference templates in $\mathrm{qPCR}$. The primers for $p c b A B$ (F-pcbAB and R-pcbAB) and $p c b C$ (F-pcbC and R$\mathrm{pcbC}$ ) (Table 2) were used to assess the cluster copy number in genomic DNA. P. chrysogenum strains DS54465 (hdfAD), Wisconsin54-1255, and DS50652 (lacking penicillin biosynthesis gene cluster) (Table 1) were used as controls containing 8,1 , and 0 penicillin gene clusters, respectively. The gene copy numbers were analyzed on a Miniopticon ${ }^{\mathrm{TM}}$ system (BioRad) using the BioRad CFX manager software in which the $C(t)$ values were determined automatically by regression. The SensiMix ${ }^{\mathrm{TM}}$ SYBR mix (Bioline, Alphen aan den Rijn, The Netherlands) was used as a master mix for qPCR with $0.4 \mu \mathrm{M}$ primers and $40 \mathrm{ng}$ gDNA in a $25-\mu \mathrm{L}$ reaction volume. The copy numbers were calculated from duplicate experiments

\section{Sequencing of DS17690 PcvelA and PclaeA alleles}

PcvelA and PclaeA genes were PCR amplified from DS17690 genomic DNA with the primers pairs veA Fw-veA Rv and lae $A$ $\mathrm{Fw}-\mathrm{lae} A \mathrm{Rv}$, respectively (Table 2 ). Genomic DNA was isolated as mentioned earlier. The PCR products were sequenced by the Sanger method at Baseclear (Leiden, The Netherlands). The resulting sequences were compared to the corresponding Wisconsin54-1255 sequences using Clustal W software (Larkin MA et al., 2007).

\section{Media and culture conditions}

$P$. chrysogenum strains were grown in a glucose-limited defined mineral medium with the following composition per liter: $0.8 \mathrm{~g} \mathrm{KH} \mathrm{KO}_{4}, 3.5 \mathrm{~g}\left(\mathrm{NH}_{4}\right)_{2} \mathrm{SO}_{4}, 0.5 \mathrm{~g} \mathrm{MgSO} \cdot 7 \mathrm{H}_{2} \mathrm{O}, 7.5 \mathrm{~g}$ glucose, and $10 \mathrm{ml}$ of trace elements solution. The trace element solution contained $15 \mathrm{~g} \cdot \mathrm{L}^{-1} \mathrm{Na}_{2} \mathrm{EDTA} \cdot 2 \mathrm{H}_{2} \mathrm{O}, 0.5 \mathrm{~g} \cdot \mathrm{L}^{-1}$ $\mathrm{CuSO}_{4} \cdot 5 \mathrm{H}_{2} \mathrm{O}, 2 \mathrm{~g} \cdot \mathrm{L}^{-1} \mathrm{ZnSO} 4 \cdot 7 \mathrm{H}_{2} \mathrm{O}, 2 \mathrm{~g} \cdot \mathrm{L}^{-1} \mathrm{MnSO}_{4} \cdot \mathrm{H}_{2} \mathrm{O}$, $4 \mathrm{~g} \cdot \mathrm{L}^{-1} \mathrm{FeSO}_{4} \cdot 7 \mathrm{H}_{2} \mathrm{O}$, and $0.5 \mathrm{~g} \cdot \mathrm{L}^{-1} \mathrm{CaCl}_{2} \cdot 2 \mathrm{H}_{2} \mathrm{O}$. Penicillin $\mathrm{G}$ production was induced by adding $0.58 \mathrm{~g} \cdot \mathrm{L}^{-1}$ of phenylacetic acid (PAA) to the medium. The necessary amount of $\mathrm{KOH}$ was added to set the medium $\mathrm{pH}$ at 5.5.

All strains were grown in aerobic, glucose-limited chemostat cultures in a 3-L bioreactor (Applikon, Schiedam, The Netherlands) at pH6.5 and at a dilution rate of $0.03 \mathrm{~h}^{-1}$ as previously described (Harris et al., 2009b). Chemostat cultures were assumed to be in steady state when, after at least 5 volume changes of continuous cultivation under the same conditions, culture dry weight, and off gas $\mathrm{CO}_{2}$ analyses differed by less than $4 \%$ over two consecutive volume changes (Daran-Lapujade et al., 2009).

\section{Analytical methods}

Biomass dry weight was measured in duplicate, by filtering $10 \mathrm{~mL}$ of culture sample over preweighted glass fiber filters (Type A/E, Pall Life Sciences, East Hills, NY). The filters were washed with demineralized water, dried in a microwave oven for $20 \mathrm{~min}$ at $600 \mathrm{~W}$, and subsequently weighted. 
A

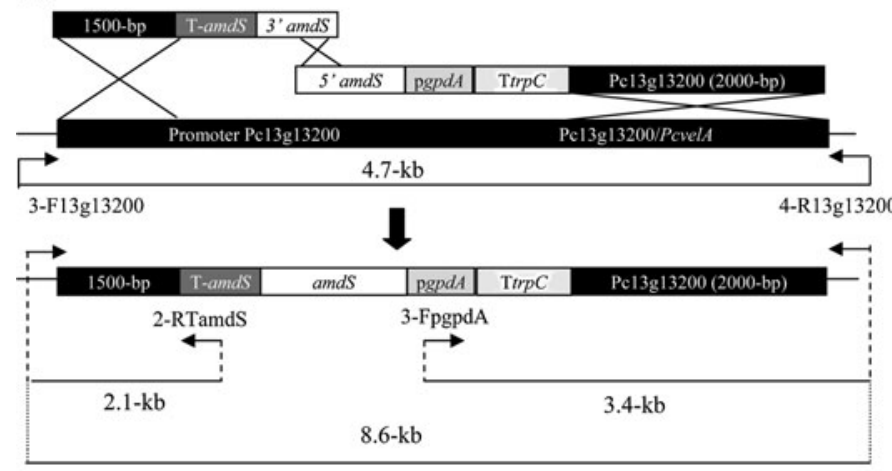

B

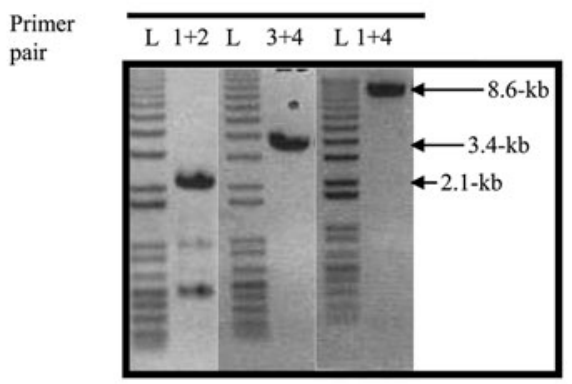

C
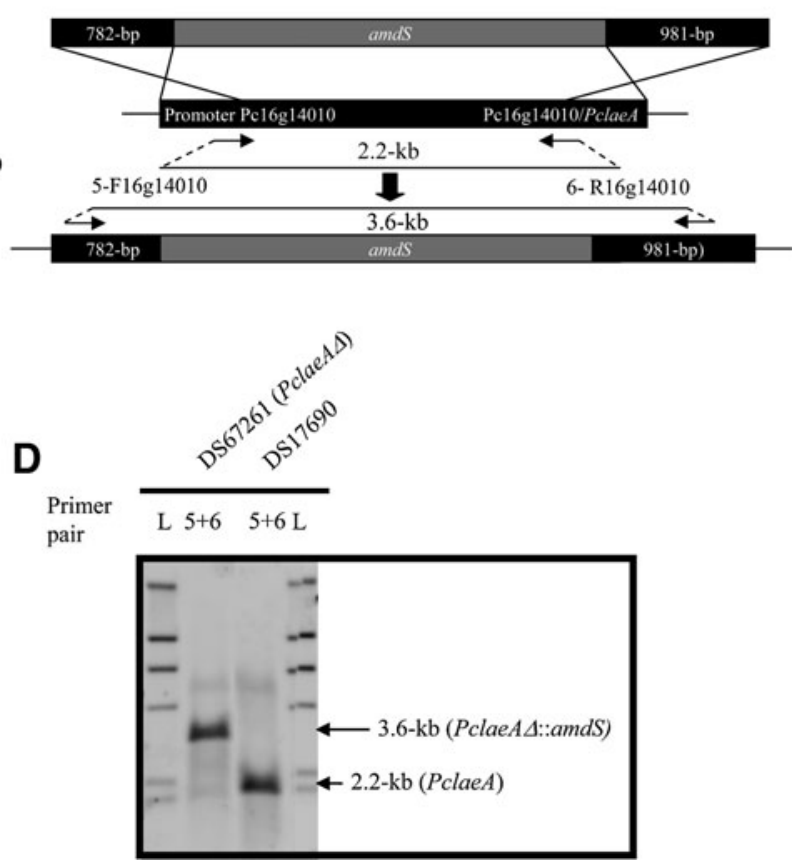

FIG. 1. Strain construction scheme. Construction and characterization of the strains DS63171 (PcvelAL/Pc13g13200) (A, B) and DS67261 (Pc16g14010PclaeAD) (C, D). (A) Schematic representation of DS63171 (Pc13g13200/PcvelAD) strain construction using bipartite vectors. The arrows represent the primer pairs used to verify the correct gene insertion, with respective fragment size. (B) PCR confirmation of PcvelA deletion and integration of amdS marker in strain DS63171 (hdfA $\Delta$ PcvelA $\Delta$ ). (C) Schematic representation of DS67261 (hdfA $\mathrm{PclaeA \Delta )}$ strain construction. The arrows represent the primer pairs used to verify the correct gene insertion, with respective fragment size. (D) PCR confirmation of amdS integration in strain DS67261 ( $h d f A \Delta$ PclaeA $\Delta$ ) and comparison to PclaeA gene in reference strain DS17690. L denotes the ladder used.

Glucose and PAA concentrations in media and culture supernatants were analyzed by HPLC (Waters Alliance 2695 Separation Module supplied with a Waters 2487 Dual Absorbance Detector and a Waters 2410 Refractive Index Detector; Waters, Milford, MA) containing a Biorad $\mathrm{HPX87H}$ column (BioRad) eluted at $60^{\circ} \mathrm{C}$ with $0.5 \mathrm{mM} \mathrm{H}_{2} \mathrm{SO}_{4}$ at a flow rate of $0.6 \mathrm{~mL} \cdot \mathrm{min}^{-1}$. Quantitative ${ }^{1} \mathrm{H}-\mathrm{NMR}$ was used to measure extracellular concentration of penicillin $\mathrm{G}, \mathrm{ACV}$, and IPN from $P$. chrysogenum cultures. Quantitative ${ }^{1} \mathrm{H}-\mathrm{NMR}$ experiments were performed at $600 \mathrm{MHz}$ on a Bruker Avance 600 spectrometer (Bruker, Wormer, The Netherlands). To a known quantity of filtrate, a known quantity of internal standard (maleic acid), dissolved in phosphate buffer, was added prior to lyophilization. The residue was dissolved in $\mathrm{D}_{2} \mathrm{O}$ and measured at $300 \mathrm{~K}$. The delay between scans (30 s) was more than five times $\mathrm{T} 1$ of all compounds, so the ratio between the integrals of the compounds of interest and the integral of the internal standard was an exact measure for the quantity of the penicillins.

\section{Transcriptome analysis}

Chemostat sampling was performed by rapidly filtering $60 \mathrm{~mL}$ of culture broth over a glass fiber filter (Type A/E, Pall Life Sciences). The filter with mycelium was immediately wrapped in aluminum foil, quenched in liquid nitrogen, and stored at $-80^{\circ} \mathrm{C}$. Samples were processed as previously described (Harris et al., 2009a; van den Berg et al., 2008). Acquisition and quantification of array images and data filtering were performed by using Affymetrix GeneChip Operating Software (GCOS version 1.2). All arrays were globally scaled to a target value of 100 , using the average signal from all genes (global scaling). The arrays were analyzed as previously described (Harris et al., 2009a). To assess statistically significant changes in expression, replicate arrays experiment were compared by using the software Significance Analysis of Microarray (SAM version 2.0) (Tusher et al., 2001). The foldchange was set to 2.5 and a maximum false discovery rate of $7 \%$ was used. Gene clusters were evaluated for enrichment in MIPS (Munich Information Center for Protein Sequences) categories (version 1.3) by employing hypergeometric distribution with a $p$-value cutoff of $10^{-3}$ (Harris et al., 2009a; Knijnenburg et al., 2009). The transcriptome data analyzed in this study have been deposited at the Genome Expression Omnibus database http://www.ncbi.nlm.nih.gov/geo/) under the accession number GSE31633. The transcriptome data of the Wisconsin54-1255 were derived from the GEO series GSE9825.

\section{Results}

\section{Velvet complex genes are conserved in Penicillium chrysogenum}

The ascomycetous fungus Penicillium chrysogenum is phylogenetically closely related to Aspergillus species (van den Berg et al., 2008; Verweij et al., 1995). Based on the extensive molecular analysis of the A. nidulans Velvet complex (Bayram 
Table 3. Similarity of Velvet Complex Ortholog Proteins in P. chrysogenum

\begin{tabular}{|c|c|c|c|c|c|}
\hline A. nidulans & P. chrysogenum & & Score & E-value & Similarity (\%) \\
\hline LreA & Pc20g08380 & & 641 & 0 & 60 \\
\hline LreB & Pc22g02540 & & 264 & $3.00^{\mathrm{E}-71}$ & 55 \\
\hline FphA & Pc06g00040 & & 1168 & 0 & 70 \\
\hline KapA & Pc21g01970 & & 1034 & 0 & 97 \\
\hline LaeA & Pc16g14010 & PcLaeA & 481 & $2.00^{\mathrm{E}-136}$ & 75 \\
\hline VeA & Pc13g13200 & PcVelA & 424 & $4.00^{\mathrm{E}-119}$ & 61 \\
\hline VelB & Pc22g22320 & & 293 & $4.00^{\mathrm{E}-80}$ & 63 \\
\hline VosA & Pc22g06890 & & 393 & $6.00^{\mathrm{E}-110}$ & 66 \\
\hline
\end{tabular}

A BlastP was performed using Aspergillus nidulans query to search translation of P. chrysogenum ORFeome (van den Berg et al., 2008).

et al., 2008; Calvo, 2008; Fischer, 2008; Kato et al., 2003; Kim et al., 2002; Sprote and Brakhage, 2007), a genome-wide search was performed on the $P$. chrysogenum protein database (comprising 13,670 proteins; Genbank accession number: AM920416 to AM920464) (van den Berg et al., 2008) for sequences orthologous to the A. nidulans Velvet complex proteins. This search yielded clear orthologs for all eight subunits of the A. nidulans Velvet complex (Table 3).

Our analysis confirmed the previously reported (Dreyer et al., 2007) absence in PcVelA (Pc13g13200) of a PEST sequence, a motif implicated in targeted protein degradation, and clear difference in the nuclear export and localization motives. The predicted protein sequence of PcLaeA (Pc16g14010) (55\% sequence identity to the $A$. nidulans LaeA amino acid sequence) exhibited four amino acids substitution in the methyltransferase domain (199-TGIWSIEVANKFPGSFV-215) compared to the $A$. nidulans sequence (positions $203 \mathrm{~S} \rightarrow \mathrm{A}, 210 \mathrm{~F} \rightarrow \mathrm{Y}, 212$ $\mathrm{G} \rightarrow \mathrm{D}$, and $213 \mathrm{~S} \rightarrow \mathrm{A}$ ).

To investigate a possible impact of classical strain improvement (Newbert et al., 1997) on PcvelA and PclaeA, the sequences of these genes were compared between the $\beta$-lactam high producing strain DS17690 and its parental strain Wisconsin54-1255. Two single nucleotide differences were identified in DS17690 PcvelA, in positions $343\left(\mathrm{C}^{\text {Wisconsin }} \rightarrow \mathrm{T}^{\mathrm{DS17690}}\right)$ and $585\left(\mathrm{C}^{\text {Wisconsin }} \rightarrow \mathrm{T}^{\mathrm{DS17690}}\right)$ and one in DS17690 PclaeA in position $1012\left(\mathrm{G}^{\text {Wisconsin }} \rightarrow \mathrm{A}^{\mathrm{DS} 17690}\right)$. However, these mutations did not alter the predicted amino acid sequences of the PcLaeA and PcVelA proteins.

\section{Construction of PcvelA and PclaeA knock-down strains}

To further investigate the role of the Velvet complex as a global regulator in penicillin-producing $P$. chrysogenum cultures, PcvelA and PclaeA mutants were constructed by protoplast transformation of strain DS54465 (DS17690 hdfAD) (Snoek et al., 2009). Correct integration of the amdS cassettes was confirmed by Southern blot (data not shown) and PCR (Fig. 1). Preliminary experiments with a PcvelA mutant in this strain background indicated drastic reduction (by ca. 90\%) of penicillin G production (data not shown). However, subsequent analysis showed that this reduced productivity coincided with the loss of all but one of its six to eight penicillin gene clusters. Previous studies have shown the instability of the number of penicillin gene clusters in high-producing strains, caused by recombination of the direct repeats in these clusters (Fierro et al., 1995; Nijland et al., 2010). Indeed, protoplast transformationassociated loss of penicillin gene cluster amplicons has been used in the construction of a "cluster-free" P. chrysogenum strain (DS50661) (Harris et al., 2009a). Construction of new PcvelAs and PclaeAs mutants in the high penicillin producing strain therefore involved a rigorous copy number evaluation of the penicillin biosynthetic genes by quantitative PCR.

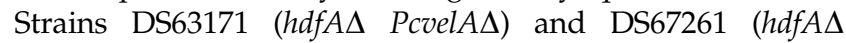
PclaeA $\Delta$ ) were therefore analyzed by q-PCR, using the strains DS54465 ( $h d f A \Delta)$ (six to eight penicillin biosynthetic clusters), Wisconsin54-1255 (one penicillin biosynthetic cluster), and DS50562 (zero penicillin biosynthetic cluster) as references. Furthermore, the comparison of the number of penicillin clusters of the strain DS54465 ( $h d f A \Delta$ ) (this study) and its ancestor DS17690 (Douma et al., 2011) did not reveal any intermediate loss of cluster between the two strains. Deletion

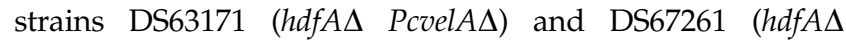
PclaeAS) did not showed significant changes in penicillin biosynthesic gene cluster number relative to DS54465 and DS17690 (Fig. 2) and therefore were used in further analysis.

\section{Impact of PcvelA and PclaeA mutations on physiology and penicillin $G$ production in glucose-limited chemostats}

To analyze the impact of the Velvet complex on $\beta$-lactam production under industrially relevant conditions, the reference strain $P$. chrysogenum DS17690, the PcvelAs strain DS63171 and the PclaeAS strain DS67261 were grown in glucose-limited chemostat cultures with and without addition of phenylacetate (PAA), side-chain precursor for penicillin $\mathrm{G}$ synthesis. The main noticeable macroscopic difference between the Velvet complex mutants and the reference strain was a higher propensity of both mutants to grow on the solid surfaces of the bio-reactor. However, microscopic analysis did not reveal a difference in hyphal morphology between the strains (data not shown).

When grown in the presence of PAA, both mutants exhibited a significant $(t$-test, $p<0.01$ ) decrease of the penicillin $G$ production rate that, however, did not exceed $30 \%$ relative to the DS17690 strain (Table 4). The decreased production of penicillin $\mathrm{G}$ was not accompanied by a reduction of the rate of PAA consumption, which was not significantly different in the mutant and reference strains ( $t$-test, $p>0.01)$. Biomass yields on glucose $\left(\mathrm{Y}_{\mathrm{SX}}\right)$ and specific rates of glucose consumption (qGlucose) did not differ significantly among the three strains. Although the titer of the penicillin intermediate isopenicillin $\mathrm{N}$, which is also produced in the absence of the side-chain precursor PAA (Harris et al., 2009a), exhibited 


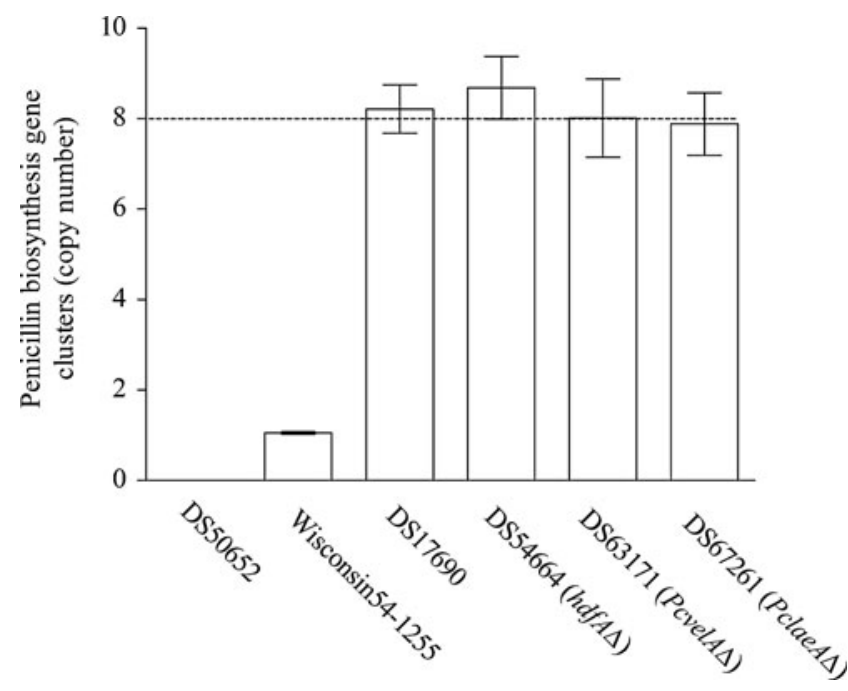

FIG. 2. Copy number of the penicillin biosynthesis cluster. Determination of the penicillin biosynthesis cluster copy number in the deletion strains $P$. chrysogenum DS63171

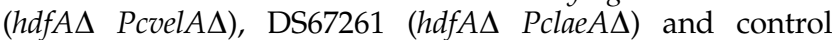
strains Wisconsin54-1255, DS54465, and DS17690. Real-time quantitative-PCR using specific primer pairs for penicillin biosynthetic genes $p c b A B$ and $p c b C$ (Table 2) were performed. Copy number data were normalized using act $A$ (encoding $\gamma$ actin). Error bars presented standard deviation from at least independent duplicate experiments.

some fluctuations, these did not reveal a clear impact of the gene deletions (Table 4).

\section{Chemostat-based transcriptome analysis: data quality and overall responses}

The relevance of the Velvet complex as a global regulator in aerobic, glucose-limited chemostat cultures was analyzed by a full transcriptome analysis on the PclaeAs, PcvelAs, and reference strains. DNA-microarray analysis was performed on mRNA isolated from glucose-limited chemostat cultures $\left(D=0.03 \mathrm{~h}^{-1}\right)$ of each of the three strains, grown in the presence and in the absence of PAA. For each strain, transcriptome analysis was performed from at least two independent replicate chemostat cultures. The average coefficient of variation for transcriptome data from replicated cultures was below $20 \%$, in accordance with previous chemostat-based transcriptome analyses in P. chrysogenum (Douma et al., 2011; Gombert et al., 2011; Harris et al., 2009a, 2009b; Koetsier et al., 2010; Snoek et al., 2009; van den Berg et al., 2008).

Four pair-wise comparisons (two mutant strains and two cultivation regimes) were executed to assess the impact of the PclaeA and PcvelA deletions on the P. chrysogenum transcriptome. This analysis identified 550 transcripts (corresponding to $4 \%$ of the entire $P$. chrysogenum genome) whose levels in the reference strain and at least one of the mutants differed under at least one condition (Fig. 3 and Supplementary Table S1). The transcript data of the Velvet complex mutants were compared to data from the DS17690 strain and not from the DS54465 ( $h d f A \Delta)$. As previously shown by Snoek et al. (2009), the difference in gene expression between the two strains was extremely small, as only 26 genes $(0.18 \%$ of the $P$. chrysogenum genes) were deemed significantly changed. Moreover we did not observe differential expression of any of the 26 genes between DS54465 ( $h d f A \Delta)$ ), DS63171 ( $h d f A \Delta$

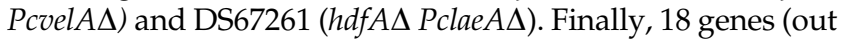
of 26) retained their differential expression relative to the DS17690. Based on these comparisons we concluded that transcript data derived from the DS17690 strain were suitable to execute the comparative analysis with the strains DS63171

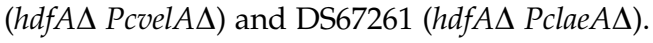

In a separate analysis, we compared our results with previously published transcript data obtained with batch cultures of P. chrysogenum PcvelA mutant in P2niaD strain background. Although Hoff and coauthors (2010) investigated the impact of PcVelA in P. chrysogenum, the two studies differed in several points. Although we analyzed the effect on

Table 4. Growth and Physiological Characteristics of P. chrysogenum Strains (DS17690, DS63171 (hdf $A \Delta$ PCVel $A \Delta)$ ANd DS67261 (hdf $A \Delta$ PClAe $A \Delta)$

\begin{tabular}{|c|c|c|c|c|c|c|c|}
\hline Strain & ${ }^{\mathrm{a}} P A A$ & ${ }^{\mathrm{b}} Y_{S X}(g / g)$ & $\begin{array}{c}{ }^{c} q_{\text {Glucose }} \\
(\mathrm{mmol} / \mathrm{g} / \mathrm{h})\end{array}$ & $\begin{array}{l}\mathrm{d}_{q_{\text {Side chain }}} \\
(\mathrm{mmol} / \mathrm{g} / \mathrm{h})\end{array}$ & $\begin{array}{l}{ }^{\mathrm{e}} q_{\text {Penicillin } G} \\
(\mathrm{mmol} / \mathrm{g} / \mathrm{h})\end{array}$ & ${ }^{\mathrm{f}} I P N(m M)$ & References \\
\hline \multirow[t]{2}{*}{ DS17690 } & - & $0.37 \pm 0.00$ & $0.43 \pm 0.01$ & NA & NA & $0.10 \pm 0.07$ & $\begin{array}{l}\text { (Harris et al., 2006), } \\
\text { this study }\end{array}$ \\
\hline & + & $0.35 \pm 0.01$ & $0.48 \pm 0.02$ & $0.024 \pm 0.002$ & $0.020 \pm 0.001$ & $0.005 \pm 0.00$ & $\begin{array}{l}\text { (Harris et al., 2006), } \\
\text { this study }\end{array}$ \\
\hline DS63171 & - & $0.39 \pm 0.01$ & $0.42 \pm 0.01$ & NA & NA & $0.03 \pm 0.04$ & This study \\
\hline$($ PcvelAs $)$ & + & $0.34 \pm 0.02$ & $0.49 \pm 0.04$ & $0.030 \pm 0.002$ & $0.016 \pm 0.001$ & $0.04 \pm 0.02$ & This study \\
\hline DS67261 & - & $0.35 \pm 0.03$ & $0.49 \pm 0.07$ & NA & NA & $0.10 \pm 0.02$ & This study \\
\hline$($ PclaeA $)$ & + & $0.35 \pm 0.01$ & $0.50 \pm 0.01$ & $0.031 \pm 0.008$ & $0.014 \pm 0.001$ & $0.02 \pm 0.01$ & This study \\
\hline
\end{tabular}

Physiological and metabolite analysis of indicated $P$. chrysogenum strains grown on glucose-limited chemostat cultivations $\left(D=0.03 \mathrm{~h}^{-1}\right.$, $\left.T=25^{\circ} \mathrm{C}, \mathrm{pH}=6.5\right)$ in the presence or absence of the side-chain precursor phenylacetate (PAA). Data are presented as averages \pm mean deviation from at least two independent replicate cultures.

a Presence or absence of phenylacetate.

${ }^{\mathrm{b}}$ Biomass yield on glucose ( $\mathrm{g}$ of biomass per $\mathrm{g}$ of glucose).

${ }^{c}$ Biomass specific glucose consumption rate (mmol of glucose consumed/g biomass per hour).

${ }^{\mathrm{d} B i o m a s s}$ specific phenylacetate consumption rate (mmol of phenylacetate consumed/g biomass per hour).

eBiomass specific penicillin $\mathrm{G}$ production rate ( $\mathrm{mmol}$ of penicillin $\mathrm{G}$ produced per $\mathrm{g}$ biomass per hour).

${ }^{\mathrm{f}}$ Extra cellular concentration of Isopenicillin $\mathrm{N}$ (mmol per liter).

NA, not applicable. 


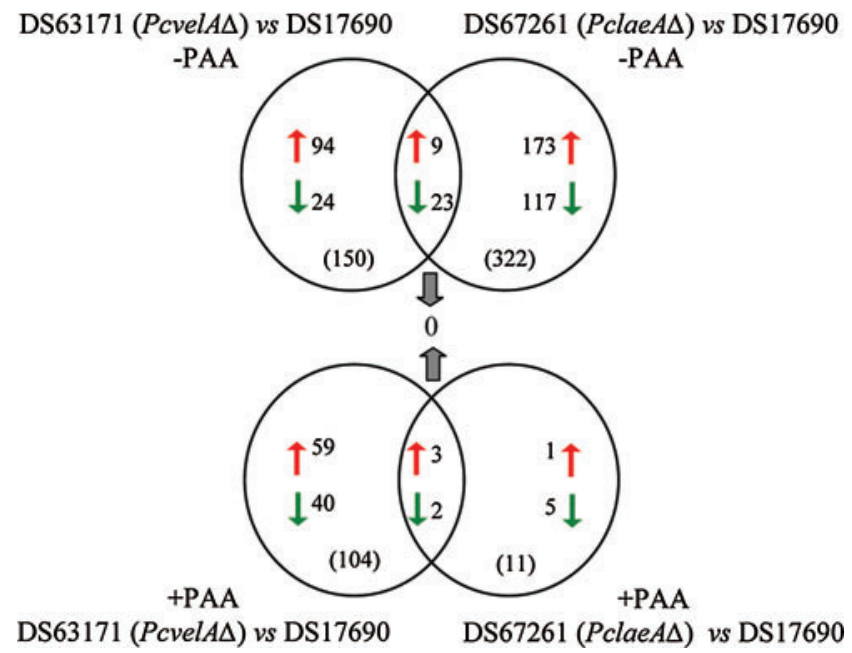

FIG. 3. Transcriptome analysis of $P$. chrysogenum strains DS63171 (hdfAS PcvelAs) and DS17690 and of DS67261 $(h d f A \Delta$ PclaeA $\Delta)$. Results of the pair-wise comparison of the

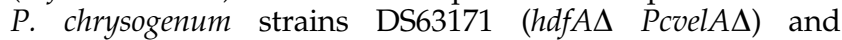
DS17690 and of DS67261 (hdfAS PclaeAs) and DS17690 in presence and absence of PAA. Circles indicate a strain grown under a specific condition. Red arrows represent the number of genes with a higher transcript level and green arrows the genes that showed a lower transcript level versus the strain DS17690. The total number of differently expressed genes is mentioned in between brackets.

cultures that produced or not penicillinG, Hoff et al. (2010), instead of PAA as a side chain, used phenoxyacetate (POA) to produce a different type of penicillin, $\mathrm{PenV}$, and compared the transcriptome in producing conditions only. In addition, the genes deemed differentially expressed by Hoff et al. (2010) were not made available (Hoff et al., 2010), Therefore we compared the set of genes that showed a different transcript level in glucose-limited chemostat cultures of the PcvelAs strain with added PAA (generated in this study) with the pairwise comparison of the 96-h time point of prolonged batch cultures of a PcvelAs mutant in the high-producing P2niaD strain background, grown in the presence of POA (Hoff et al., 2010) (|fold change $\mid>2.5$ ). Strikingly, less than $1 \%$ of the responsive genes were found to be common to the two studies (Fig. 4; Supplementary Table S2).

\section{Transcriptional coresponses to PcvelA and PclaeA deletions}

Because PcVelA and PcLaeA are both core components of the Velvet complex, we compared the transcriptome of the PclaeAs and PcvelA $\Delta$ mutants derived from biomass samples grown in glucose-limited chemostat cultures. Only a small fraction $(7 \%, 37 / 550)$ of the transcriptional response was shared between the two deletion mutants (Fig. 3). With the exception of the acetamidase ( $a m d S$ ) deletion marker and the gene Pc22g15510 ( $h d f A$ ), which was deleted in the strain in which the deletions were introduced (Snoek et al., 2009), no genes were differently expressed in all four two-way comparisons. The amdS gene was not taken into account in further analysis as it is not a $P$. chrysogenum native gene (Table 5). A closer look at the expression signal of both PclaeA and PcvelA confirmed the absence of transcript and eventually the correct deletion in the strains DS67261 (hdfA $\Delta$ PclaeA $\Delta)$ and DS63171 (hdfA $\Delta$ PcvelA $\Delta$ ), respectively.

A Fisher Exact test did not reveal any significant enrichment of functional categories among the remaining 550 differentially regulated genes. 23 genes were downregulated in both mutants relative to the reference strain in cultures that were grown in the absence of added PAA (Table 5). Of this gene set 11 belong to two groups that showed chromosomal positional linkage. The first group comprised six genes (Pc06g02040-Pc06g02100) of unknown function. The second set of five linked genes (Pc12g06310-Pc12g06370) included genes that, according to their annotation, are likely to be involved in secondary metabolism (Keller et al., 2005). The protein Pc12g06310 shared very high similarity (97\%) with the Ari1 from Penicillium roqueforti, an aristolochene synthase (Proctor and Hohn, 1993). Aristolochene is the first intermediate in the synthesis of a sesquiterpene pathogenesis-related toxin, for which the complete biosynthetic pathway remains unidentified.

Two other genes displayed interesting annotations. Pc16g12580 shared high similarity to a blue-light receptor and was upregulated strongly in both deletion strains relative to the reference strain (3.1- and 9.9-fold change for PcvelA $\Delta$ and $P$ clae $A \Delta$, respectively) when grown in the absence of PAA. In A. nidulans, the VelB/VeA/LaeA complex connects light to developmental regulation and control of secondary metabolism (Bayram et al., 2008; Fischer, 2008; Sprote and Brakhage, 2007; Sarikaya et al., 2010; Stinnett et al., 2007). Upregulation of Pc16g12580 might therefore be involved in some compensatory response to the disruption of the integrity of the Velvet complex. Finally, in presence of PAA the putative regulatory gene Pc21g08860 (that exhibits strong similarity to acv $A$ gene expression regulator Pc804 like protein) was upregulated 3.6fold (Table 5), which suggests that a negative effect of the inactivation of the Velvet complex may be partially compensated for by another regulatory mechanism.

\section{Impact of PclaeA and PcvelA mutations on expression of secondary metabolism genes}

To further assess the impact of the PcvelA and PclaeA deletions on regulation of secondary metabolism in glucoselimited chemostat cultures of $P$. chrysogenum, we specifically analyzed the transcript profiles of genes that, based on sequence similarity and/or functional analysis data, can be implicated in secondary metabolism. This targeted analysis also included the eight $P$. chrysogenum Velvet complex orthologs (Table 3). None of the eight Velvet complex orthologs (with the obvious exception of the deleted gene itself) showed drastic transcriptional changes in the deletion strains, irrespective of the inclusion of PAA in the growth media (Fig. 5). To investigate whether the intensive strain improvement program from which $P$. chrysogenum DS17690 originates might have affected expression of these genes, transcript levels of Velvet complex genes, including PcvelA and PclaeA were compared in chemostat cultures to the ancestor strain Wisconsin54-1255. This analysis did not provide evidence for changes in the expression levels of Velvet genes during strain improvement (Fig. 5).

During the first analysis of the $P$. chrysogenum genome sequence (van den Berg et al., 2008), 51 genes were annotated as nonribosomal peptide synthases (NRPS), polyketide synthases 


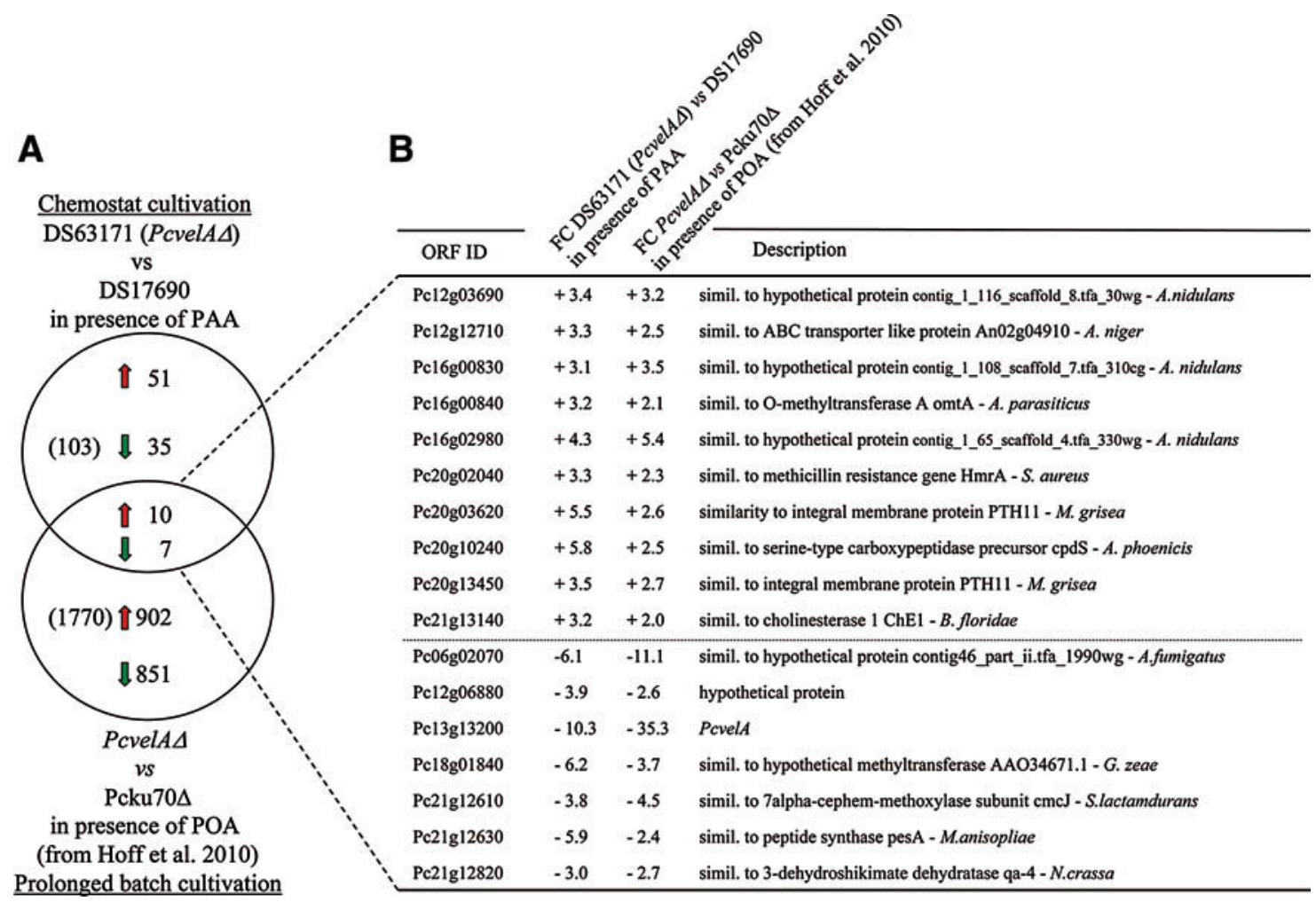

FIG. 4. Comparative transcript analysis of P. chrysogenum PcvelA mutants in prolonged batch and chemostat cultivations. Comparison of genes that showed at least twofold change in prolonged batch cultivations with POA of PcvelA $\Delta$ versus Pcku70 as reported in Hoff et al. (2010) with genes that showed differential expression in glucose chemostat cultures of DS63171 (PcvelA $\Delta)$ relative to the reference DS17690 as reported in this study. The fold changes from the data reported in Hoff et al. (2010) were calculated by comparing the 96-h points in the PcvelA $\Delta$ and Pcku70 $\Delta$. Red arrows represent the number of genes with a higher transcript level in the PcvelA $\Delta$ mutant than in its respective reference and green arrows represent the genes that showed a lower transcript level in the PcvelA $\Delta$ mutant than in its respective reference. The total number of differently expressed genes is mentioned in between brackets.

(PKS), NRPS-like enzymes, PKS-like enzymes, and NRPS-PKS hybrids. The paradigm of NRPS in P. chrysogenum is the gene $a c v A(p c b A B)$ that encodes for $\delta$-(L- $\alpha$-aminoadipyl)-L-cysteineD-valine synthetase, which catalyzes the first step of penicillin biosynthesis. In both mutants, irrespective of the presence of PAA, expression of $a c v A$ was not substantially altered, consistent with the small reduction of penicillin $G$ productivity in the PcvelA and PclaeA mutants (Table 4). Similarly, expression of the other two clustered genes necessary for penicillin $\mathrm{G}$ synthesis, isopenicillin $\mathrm{N}$ synthase $(p c b C)$ and isopenicillin $\mathrm{N}$ acyltransferase (penDE) was not strongly affected by the PcvelA and PclaeA deletions (Fig. 5). This result is in total contrast with the previous studies in which the deletions of PcvelA and PclaeA were accompanied with a strong downregulation of not only $p c b A B$ but also pen $D E$ and $p c b C$.

Of the NRPS (-like), PKS (-like), and NRPS-PKS hybrids that are not involved in penicillin $G$ biosynthesis, a large fraction (34\%, 17 genes) were not expressed in any of the cultures. Another 54\% (27 genes) were not differentially expressed, leaving only six genes (12\%) (one NRPS, four PKS, and one NRPS-like) with differential expression in at least one pair wise comparison between either the PcvelA and PclaeA mutant and the DS17690 reference strain. These six genes did not exhibit consistent profiles as four were downregulated in one of the mutants while the other two were upregulated (Fig. 6).

\section{Discussion}

Inactivation of the Velvet complex genes PcvelA and PclaeA has previously been shown to have a drastic effect on penicillin production by $P$. chrysogenum. In two different $P$. chrysogenum strains, deletion of PcvelA and/or PclaeA resulted in a massive reduction of penicillin production and a strong downregulation of the expression of genes involved in penicillin biosynthesis (Hoff et al., 2010; Kosalkova et al., 2009). Whereas the two previous studies were performed in prolonged batch cultures, the present study was performed in aerobic, glucose-limited cultures at a fixed specific growth rate of $0.03 \mathrm{~h}^{-1}$, which is near to the optimum for penicillin $\mathrm{G}$ productivity in the strain background used (Hasegawa et al., 1985). Under these conditions, deletion of either PclaeA or PcvelA had only a moderate effect on penicillin production. Moreover, we did not observe the strong downregulation of penicillin biosynthesis genes and morphological changes reported previously for prolonged batch cultures (Hoff et al., 2010; Kosalkova et al., 2009). This main discrepancy could not be explained by mutations because the sequence analysis revealed no difference between the amino acid sequences of 
Table 5. Transcript Analysis of P. Chrysogenum DS17690, DS63171 (hdfA $\Delta$ PCVel $A \Delta$ ) AND DS67261 (hDFA $\triangle$ PCLAe $A \Delta)$ STRAins

\begin{tabular}{|c|c|c|c|}
\hline & Absence of phenylacetate (PAA) & Fold change & rsus DS17690 \\
\hline $\begin{array}{l}\text { Genes upreg } \\
\text { DS17690 } \\
(9 \text { genes) }\end{array}$ & $\begin{array}{l}\text { lated in both DS63171 (PcvelA } \Delta) \text { and in DS67261 (PclaeA } \Delta) \text { relative to } \\
\text { Similarity to }\end{array}$ & $\begin{array}{l}\text { DS63171 } \\
\quad(\text { PcvelA } \Delta)\end{array}$ & $\begin{array}{l}\text { DS67261 } \\
\quad(\text { PclaeA } \Delta)\end{array}$ \\
\hline Pc06g00230 & hypothetical protein contig61.tfa_1250cg-A. fumigatus & 12.0 & 4.1 \\
\hline Pc16g12580 & hypothetical blue-light-inducible protein bli-3-N. crassa & 3.1 & 9.9 \\
\hline Pc21g01220 & alcohol dehydrogenase (NADP+) ALR—S. scrofa & 3.5 & 14.7 \\
\hline Pc21g21440 & hypothetical protein & 3.8 & 4.5 \\
\hline Pc22g06430 & hypothetical protein $\mathrm{B} 5 \mathrm{O} 22.140-N$. crassa & 3.4 & 6.5 \\
\hline Pc22g07910 & hypothetical protein YGR086c-S. cerevisiae & 5.5 & 11.4 \\
\hline Pc22g13930 & glutathione-dependent formaldehyde dehydrogenase FDH - M. marinus & 5.0 & 24.1 \\
\hline Pc23g00530 & ankyrin repeat protein E4_2—Synthetic construct & 3.3 & 4.1 \\
\hline Pc49g00070 & hypothetical protein-P. marneffei & 3.4 & 20.9 \\
\hline $\begin{array}{r}\text { Genes down } \\
\text { to DS1769 }\end{array}$ & $\begin{array}{l}\text { egulated in both DS63171 (PcvelA } \Delta) \text { and in DS67261 (PclaeA } \Delta) \text { relative } \\
\text { (23 genes) }\end{array}$ & & \\
\hline Pc06g02040 & hypothetical protein 15_scaffold_1.tfa_520cg-F. graminearum & -3.6 & -10.0 \\
\hline Pc06g02050 & hypothetical protein An08g11990-A. niger & -6.3 & -17.1 \\
\hline Pc06g02070 & hypothetical protein contig46_part_ii.tfa_1990wg-A. fumigatus & -10.8 & -11.4 \\
\hline Pc06g02080 & hypothetical protein An06g02320—A. niger & -5.2 & -6.3 \\
\hline Pc06g02080 & hypothetical protein An06g02320-A. niger & -6.7 & -6.6 \\
\hline Pc06g02090 & hypothetical protein & -5.9 & -6.0 \\
\hline Pc06g02100 & hypothetical protein & -6.3 & -3.2 \\
\hline Pc12g02730 & vegetative incompatibility factor het-e1-P. anserina & -3.3 & -4.6 \\
\hline Pc12g06310 & aristolochene synthase Ari1—P. roqueforti & -7.4 & -4.5 \\
\hline Pc12g06320 & hydroquinone oxidase mcrA-S. lavendulae & -4.8 & -3.6 \\
\hline Pc12g06330 & hypothetical short chain alcohol dehydrogenase cta-C. sativus & -5.7 & -3.4 \\
\hline Pc12g06340 & hypothetical cytochrome P450 monooxygenase p450-4-G. fujikuroi & -3.5 & -3.5 \\
\hline Pc12g06370 & trichothecene 3-O-acetyltransferase TRI101-F. sporotrichioides & -4.9 & -4.0 \\
\hline Pc12g08290 & antifungal protein precursor paf- $P$. chrysogenum & -5.4 & -22.6 \\
\hline Pc13g08150 & hypothetical protein An04g03190-A. niger & -3.2 & -6.2 \\
\hline Pc20g07120 & hypothetical protein An06g00470-A. niger & -3.5 & -4.3 \\
\hline Pc20g13730 & hypothetical protein An01g11500-A. niger & -4.2 & -19.8 \\
\hline Pc20g13740 & hypothetical protein BAB55393.1-H. sapiens & -3.5 & -14.2 \\
\hline Pc21g01210 & hypothetical amine transporter SPCC18.02-S. pombe & -3.5 & -12.4 \\
\hline Pc21g22240 & cholesterol 7alpha-monooxygenase CYP7—O. cuniculus & -3.0 & -7.5 \\
\hline Pc22g15510 & ATP-dependent DNA helicase II subunit Ku70—M. musculus & -8.8 & -8.8 \\
\hline Pc22g17420 & hypothetical protein contig_1_153_scaffold_12.tfa_500cg-A. nidulans & -3.0 & -7.6 \\
\hline Pc22g21310 & hypothetical protein 192_scaffold_1.tfa_150wg-F. graminearum & -4.9 & -9.6 \\
\hline Presence of & henylacetate (PAA) & Fold change & ersus DS17690 \\
\hline $\begin{array}{r}\text { Genes upreg } \\
\text { to DS1769 }\end{array}$ & $\begin{array}{l}\text { lated in both DS63171 (PcvelA } \Delta \text { ) and in DS67261 (PclaeA } \Delta \text { ) relative } \\
\text { (3 genes) }\end{array}$ & $\begin{array}{l}\text { DS63171 } \\
(\text { PcvelA } \Delta)\end{array}$ & $\begin{array}{l}\text { DS67261 } \\
\quad(\text { PclaeA } \Delta)\end{array}$ \\
\hline$\overline{P c 06 g 00720}$ & cellulosomal scaffoldin anchoring protein $C-A$. cellulolyticus & 6.6 & 3.7 \\
\hline Pc13g10790 & hypothetical protein An16g03760-Aspergillus niger & 3.4 & 3.5 \\
\hline Pc21g08860 & acvA gene expression regulator Pc804 like protein An13g01050-A. niger & 3.6 & 2.9 \\
\hline $\begin{array}{l}\text { Genes down } \\
\text { in DS6726 }\end{array}$ & $\begin{array}{l}\text { egulated in both DS63171 (PcvelA } \Delta) \text { and } \\
(\text { PclaeA } \Delta) \text { relative to DS17690 ( } 2 \text { genes) }\end{array}$ & & \\
\hline Pc20g01520 & hypothetical protein $\mathrm{mg} 07210.1-\stackrel{\mathrm{M}}{\mathrm{M}}$ grisea & -4.2 & -3.4 \\
\hline Pc22g15510 & ATP-dependent DNA helicase II subunit Ku70—M. musculus & -7.7 & -7.7 \\
\hline
\end{tabular}

Genes with consistent, significantly different transcript levels in comparisons of PcvelA and PclaeA deletion mutants with the reference strain P. chrysogenum DS17690, grown in glucose-limited cultures in the presence and absence of PAA. These genes represent the overlap of the different pair-wise comparisons in Figure 3. 


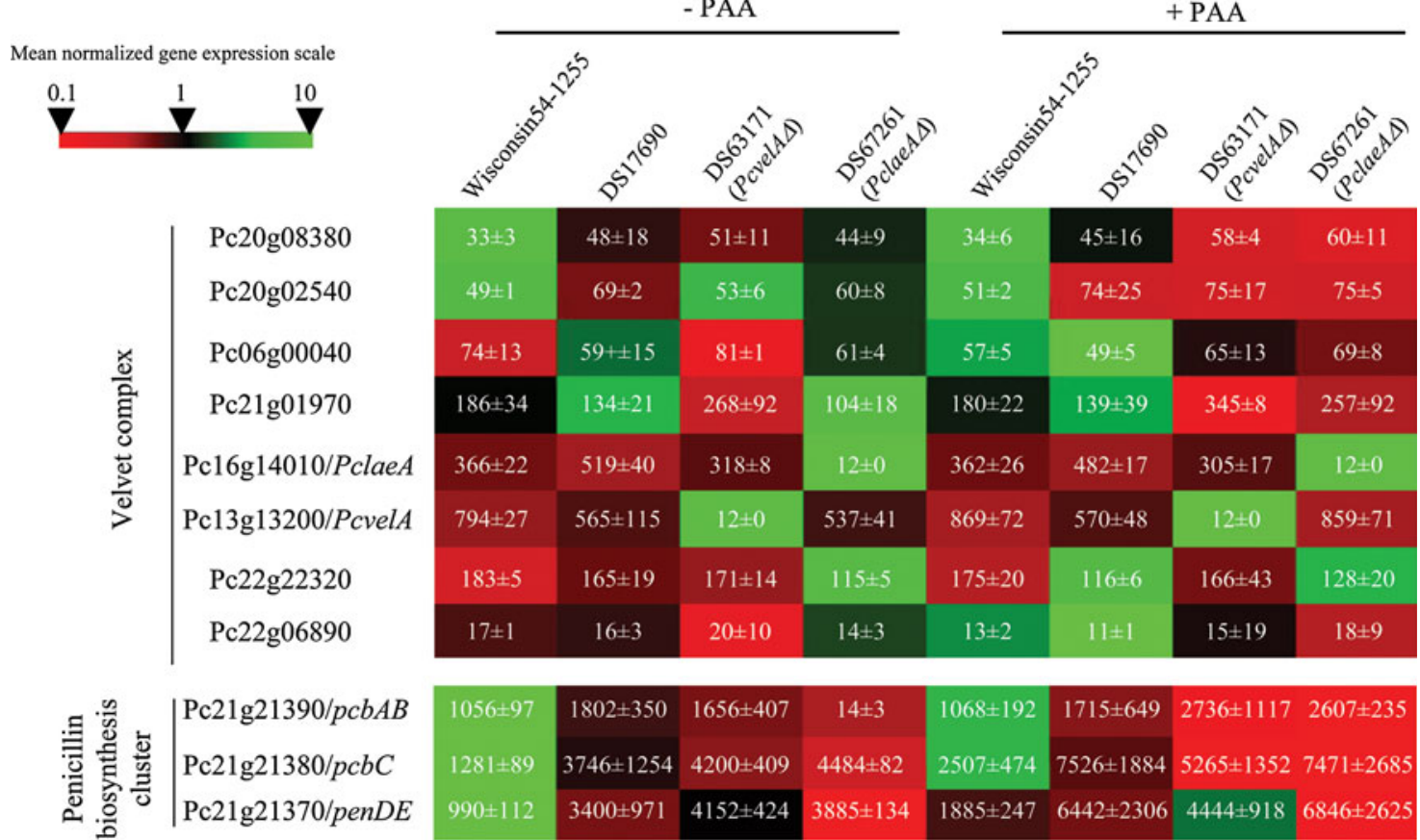

FIG. 5. I. Expression value of the genes encoding subunits of the Velvet complex and the penicillin biosynthetic enzymes in

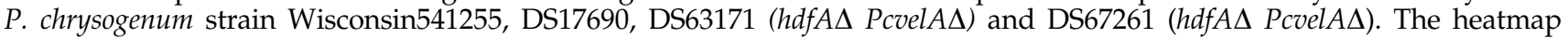
represents the item-wise Z-normalized expression value of the gene transcript levels. The numerical data represent the average hybridization intensity values of the corresponding gene probe sets on the Affymetrix array DSM_PENa520255F. Values represent the mean $\pm S D$ of data from at least duplicate experiments.

PclaeA and PcvelA from the different strains tested in the compared studies. Our results could indicate that, although the Velvet complex can act as a key regulator of penicillin biosynthesis, this role is strongly context dependent.

Secondary metabolites are characteristically produced during and after the shift from exponential growth to stationary phase, and it appears that it is under these conditions that the Velvet complex exerts its main effect on penicillin biosynthesis. Indeed, Hoff et al. (2010) showed a much stronger and wider impact of the Velvet complex on transcriptional regulation during the later phases of prolonged batch cultures (presumably corresponding with the stationary phase) than during the exponential growth phase. The present study indicates that, even at a low specific growth rate and under glucose-limited conditions, the role of the Velvet complex is much less pronounced than under essentially nongrowing conditions.

The small impact of the PclaeA and PcvelA deletions in glucose-limited, aerobic chemostat cultures indicates that the Velvet complex only plays a limited role in the activation of penicillin biosynthesis under industrially relevant conditions. This raises the question which other factor(s) contribute(s) to the efficient activation of penicillin biosynthesis under industrial conditions. A recent study in $A$. nidulans identified a multicopy suppressor of an inactive Velvet complex when overexpression of $r s m A$ was shown to restore activation of sterigmatocystin biosynthetic genes (Shaaban et al., 2010). The gene $\operatorname{rsm} A$ encodes a putative bZIP transcription factor. We therefore investigated whether a P. chrysogenum rsmA ortholog might be involved in activation of penicillin biosynthesis. Open reading frame Pc12g16510 was found to share $65 \%$ similarity with $\operatorname{rsmA}$. However, transcript levels of Pc12g16510 were sevenfold higher in P. chrysogenum Wisconsin54-1255 than in the penicillin high-producing strain DS17690, which argues against a role as activator of penicillin biosynthetic genes. Moreover, Pc12g16510 was not upregulated in the PclaeA and PcvelA mutants.

Further analysis of the transcriptome data suggested another interesting candidate for Velvet-independent activation of penicillin biosynthesis genes. The gene Pc21g08860 was upregulated in glucose-limited chemostat cultures of PcvelA and PclaeA mutants relative to the reference strain DS17690 (3.6- and 2.9-fold, respectively) but only in cultures supplemented with the penicillin $G$ side-chain precursor PAA. Pc21g08860 is identical to the $P$. chrysogenum gene labeled Pc804, previously implicated in activation of penicillin biosynthesis via its binding to the acv A promoter (Cali et al., 2004, 2005). Further analysis will reveal the exact role of Pc21g08860 in the regulation of penicillin production.

\section{Conclusion}

The present study presents a clear indication for the context dependency of transcriptional regulation of secondary metabolism in P. chrysogenum and, in particular, the role of the Velvet complex in this process. Transcriptome analysis of reference and mutant strains under a much wider range of different nutrient limitation regimes in chemostat cultures may contribute to the intellectually challenging and economically relevant goal of defining and, ultimately, controlling the role of the Velvet complex and other regulators in secondary metabolite production in P. chrysogenum and other filamentous fungi. 


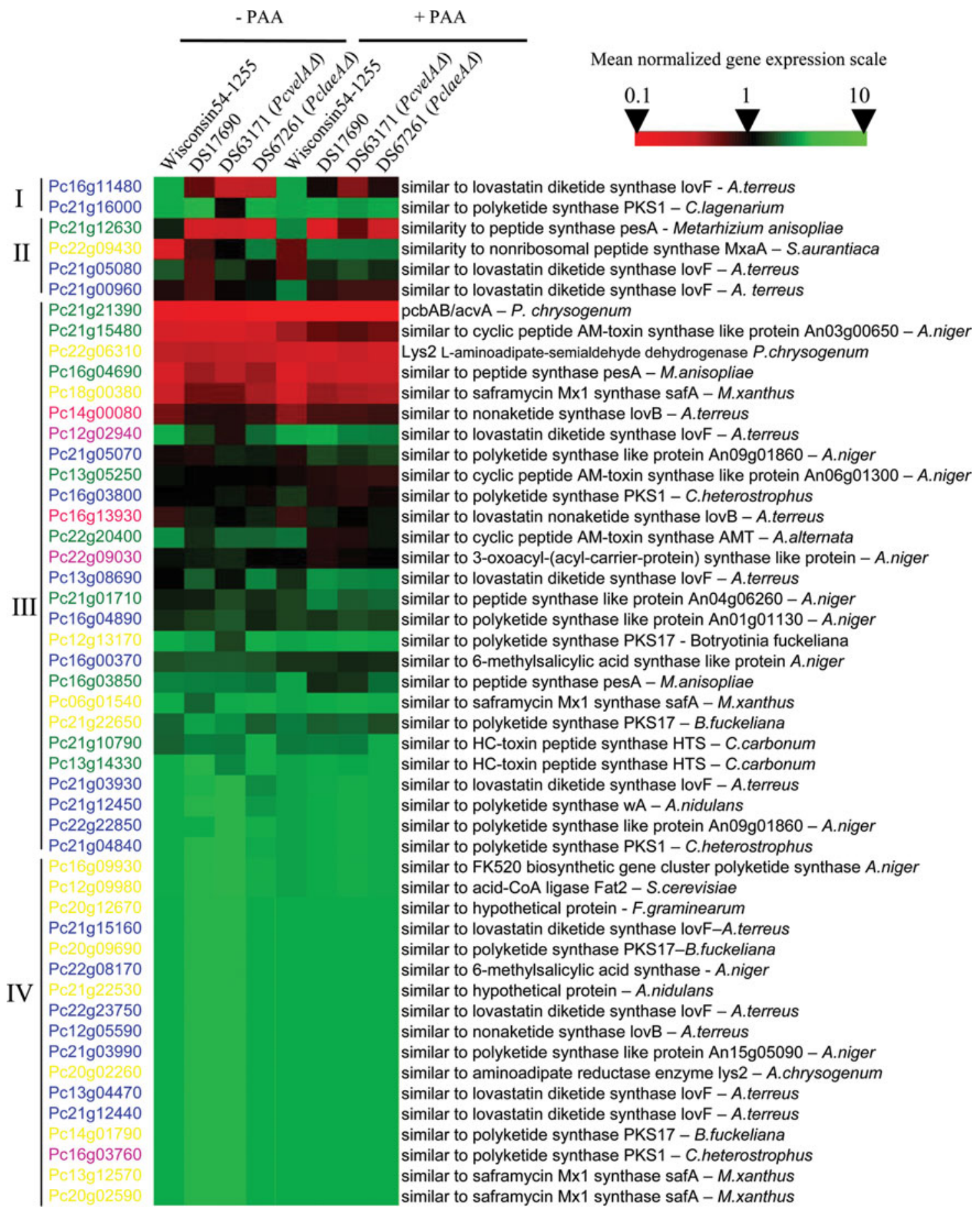

FIG. 6. Expression of secondary metabolism genes. Heat map of transcript levels of genes encoding NRPS (and -like), PKS

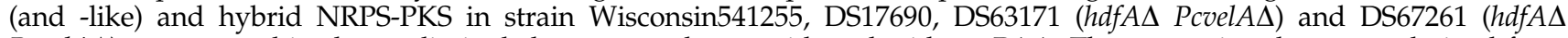
PcvelAD) grown aerobic glucose-limited chemostat cultures with and without PAA. The expression data were derived from hybridization intensity of Affymetrix DNA microarray (DSM PENa520255F). The transcript data were experiment wise Znormalized. Genes are grouped accordingly to their expression profile when compared to the strain DS17690 in upregulated $(\mathrm{FC}>2)[\mathrm{I}]$, downregulated (FC $<-2)[\mathrm{II}]$, not changed $(-2<\mathrm{FC}<2)[\mathrm{III}]$, and below detection (expression level lower than 12$)$ [IV]. Secondary metabolism group are indicated by the following code: NRPS are indicated in green, the PKS in purple, the NRPS-like in yellow, the PKS-like in blue and the RPS-PKS hybrid in pink. 


\section{Acknowledgments}

We acknowledge the financial support from the Netherlands Organization for Scientific Research (NWO) via the IBOS (Integration of Biosynthesis and Organic Synthesis) Programme of Advanced Chemical Technologies for Sustainability (ACTS) (project nr: IBOS 053.63.011).

\section{Author Disclosure Statement}

The authors declare that no conflicting financial interests exist.

\section{References}

Bayram, O., Krappmann, S., Ni, M., Bok, J.W., Helmstaedt, K., Valerius, O., et al. (2008). VelB/VeA/LaeA complex coordinates light signal with fungal development and secondary metabolism. Science 320, 1504-1506.

Bok, J.W., Chiang, Y.M., Szewczyk, E., Reyes-Dominguez, Y., Davidson, A.D., Sanchez, J.F., et al. (2009). Chromatin-level regulation of biosynthetic gene clusters. Nat Chem Biol 5, 462-464.

Cali, B., Holtzman, D., Madden, K., Milne, G., Sherman, A., Silva, J., et al. Isolated nucleic acid molecule encoding a regulator of fungal gene expression. 1-290. US6,806,082. (2004).

Cali, B., Holtzman, D., Madden, K., Milne, G., Sherman, A., Silva, J., et al. Novel regulators of fungal genes. 1-151. US2005/0095633. (2005).

Calvo, A.M. (2008) The VeA regulatory system and its role in morphological and chemical development in fungi. Fungal Genet Biol 45, 1053-1061.

Chiou, C.H., Miller, M., Wilson, D.L., Trail, F., and Linz, J.E. (2002). Chromosomal location plays a role in regulation of aflatoxin gene expression in Aspergillus parasiticus. Appl Environ Microbiol 68, 306-315.

Daran-Lapujade, P., Daran, J.M., van Maris, A.J., de Winde, J.H., and Pronk, J.T. (2009). Chemostat-based micro-array analysis in baker's yeast. Adv Microb Physiol 54, 257-311.

Demain, A., and Fang, A. (2000). The natural functions of secondary metabolites. In History of Modern Biotechnology I. A. Fiechter, ed (Springer, Berlin), pp. 1-39.

Demain, A.L., and Adrio, J.L. (2008). Contributions of microorganisms to industrial biology. Mol Biotechnol 38, 41-55.

Douma, R.D., Batista, J.M., Touw, K.M., Kiel, J.A., Krikken, A.M., Zhao, Z., et al. (2011). Degeneration of penicillin production in ethanol-limited chemostat cultivations of Penicillium chrysogenum: a systems biology approach. BMC Syst Biol 5, 132.

Dreyer, J., Eichhorn, H., Friedlin, E., Kurnsteiner, H., and Kuck, U. (2007). A homologue of the Aspergillus velvet gene regulates both cephalosporin $\mathrm{C}$ biosynthesis and hyphal fragmentation in Acremonium chrysogenum. Appl Environ Microbiol 73, 3412-3422.

Fierro, F., Barredo, J.L., Diez, B., Gutierrez, S., Fernandez, F.J., and Martin, J.F. (1995). The penicillin gene cluster is amplified in tandem repeats linked by conserved hexanucleotide sequences. Proc Natl Acad Sci USA 92, 6200-6204.

Fischer,R. (2008). Developmental biology: sex and poison in the dark. Science 320, 1430-1431.

Fox, E.M., and Howlett, B.J. (2008). Secondary metabolism: regulation and role in fungal biology. Curr Opin Microbiol 11, 481-487.

Galagan, J.E., Calvo, S.E., Cuomo, C., Ma, L.J., Wortman, J.R., Batzoglou, S., et al. (2005). Sequencing of Aspergillus nidulans and comparative analysis with A. fumigatus and A. oryzae. Nature 438, 1105-1115.

Gombert, A.K., Veiga, T., Martinez, M.P., Lamboo, F., Nijland, J.G., Driessen, A.J., et al. (2011). Functional characterization of the oxaloacetase encoding gene and elimination of oxalate formation in the beta-lactam producer Penicillium chrysogenum. Fungal Genet Biol.

Harris, D.M., Diderich, J.A., van der Krogt, Z.A., Luttik, M.A., Raamsdonk, L.M., Bovenberg, R.A., et al. (2006). Enzymic analysis of NADPH metabolism in beta-lactam-producing Penicillium chrysogenum: presence of a mitochondrial NADPH dehydrogenase. Metabol Eng 8, 91-101.

Harris, D.M., van der Krogt, Z.A., van Gulik, W.M., van Dijken, J.P., and Pronk, J.T. (2007). Formate as an auxiliary substrate for glucose-limited cultivation of Penicillium chrysogenum: impact on penicillin $G$ production and biomass yield. Appl Environ Microbiol 73, 5020-5025.

Harris, D.M., van der Krogt, Z.A., Klaassen, P., Raamsdonk, L.M., Hage, S., van den Berg, M.A., et al. (2009a). Exploring and dissecting genome-wide gene expression responses of Penicillium chrysogenum to phenylacetic acid consumption and penicillinG production. BMC Genomics 10, 75.

Harris, D.M., Westerlaken, I., Schipper, D., van der Krogt, Z.A., Gombert, A.K., Sutherland, J., et al. (2009b). Engineering of Penicillium chrysogenum for fermentative production of a novel carbamoylated cephem antibiotic precursor. Metabol Eng 11, 125-137.

Hasegawa, S., Shimizu, K., Kobayashi, T., and Matsubara, M. (1985). Efficiency of repeated batch penicillin fermentation using two fermentors. Computer simulation and optimisation. J Chem Technol Biotechnol 35, 33-42.

Hoff, B., Kamerewerd, J., Sigl, C., Mitterbauer, R., Zadra, I., Kurnsteiner, H., and Kuck, U. (2010). Two components of a velvet-like complex control hyphal morphogenesis, conidiophore development, and penicillin biosynthesis in Penicillium chrysogenum. Eukaryotic Cell 9, 1236-1250.

Horwood, P.F., Burgess, G.W., and Oakey, H.J. (2004). Evidence for non-ribosomal peptide synthetase production of cereulide (the emetic toxin) in Bacillus cereus. FEMS Microbiol Lett 236, 319-324.

Kato, N., Brooks, W., and Calvo, A.M. (2003). The expression of sterigmatocystin and penicillin genes in Aspergillus nidulans is controlled by $v e A$, a gene required for sexual development. Eukaryotic Cell 2, 1178-1186.

Keller, N.P., Turner,G., and Bennett, J.W. (2005) Fungal secondary metabolism - from biochemistry to genomics. Nat Rev Microbiol 3, 937-947.

Kim, H., Han, K., Kim, K., Han, D., Jahng, K., and Chae, K. (2002) The veA gene activates sexual development in Aspergillus nidulans. Fungal Genet Biol 37, 72-80.

Knijnenburg, T.A., Daran, J.M., van den Broek, M.A., DaranLapujade, P.A., de Winde, J.H., Pronk, J.T., et al. (2009). Combinatorial effects of environmental parameters on transcriptional regulation in Saccharomyces cerevisiae: a quantitative analysis of a compendium of chemostat-based transcriptome data. BMC Genomics 10, 53-73.

Koetsier, M.J., Gombert, A.K., Fekken, S., Bovenberg, R.A., van den Berg, M.A., Kiel, J.A., et al. (2010). The Penicillium chrysogenum aclA gene encodes a broad-substrate-specificity acylcoenzyme A ligase involved in activation of adipic acid, a side-chain precursor for cephem antibiotics. Fungal Genet Biol 47, 33-42.

Kolar, M., Punt, P.J., van den Hondel, C.A., and Schwab, H. (1988). Transformation of Penicillium chrysogenum using 
dominant selection markers and expression of an Escherichia coli lacZ fusion gene. Gene 62, 127-134.

Kosalkova, K., Garcia-Estrada, C., Ullan, R.V., Godio, R.P., Feltrer, R., Teijeira, F., et al. (2009). The global regulator LaeA controls penicillin biosynthesis, pigmentation and sporulation, but not roquefortine $\mathrm{C}$ synthesis in Penicillium chrysogenum. Biochimie 91, 214-225.

Krappmann, S., Bayram, O., and Braus, G.H. (2005). Deletion and allelic exchange of the Aspergillus fumigatus veA locus via a novel recyclable marker module. Eukaryotic Cell 4, 1298-1307.

Larkin, M.A., Blackshields, G., Brown, N.P., Chenna, R., McGettigan, P.A., McWilliam, H., et al. (2007). Clustal W and Clustal X version 2.0. Bioinformatics 23, 2948.

Luckner,M. (1991). Secondary Metabolism in Microorganisms, Plants and Animals (Springer, Berlin).

MacDonald, K.D., Hutchinson, J.M., and Gillett, W.A. (1964). Properties of heterozygous diploids between strains of Penicillium chrysogenum selected for high penicillin yield. Antonie Van Leeuwenhoek 30, 209-224.

Manzoni, M., and Rollini, M. (2002). Biosynthesis and biotechnological production of statins by filamentous fungi and application of these cholesterol-lowering drugs. Appl Microbiol Biotechnol 58, 555-564.

Menezes, J.C., Alves, S.S., Lemos, J.M., and de Azevedo, S.F. (1994). Mathematical modelling of industrial pilot-plant penicillin-G fed-batch fermentations. J Chem Technol Biotechnol 61, 123-138.

Newbert, R.W., Barton, B., Greaves, P., Harper, J., and Turner, G. (1997). Analysis of a commercially improved Penicillium chrysogenum strain series: involvement of recombinogenic regions in amplification and deletion of the penicillin biosynthesis gene cluster. J Indust Microbiol Biotechnol 19, 18-27.

Nijland, J.G., Ebbendorf, B., Woszczynska, M., Boer, R., Bovenberg, R.A., and Driessen, A.J. (2010). Nonlinear biosynthetic gene cluster dose effect on penicillin production by Penicillium chrysogenum. Appl Environ Microbiol 76, 7109-7115.

Palmer, J.M., and Keller, N.P. (2010). Secondary metabolism in fungi: does chromosomal location matter? Curr Opin Microbiol 13, 431-436.

Proctor, R.H., and Hohn, T.M. (1993). Aristolochene synthase. Isolation, characterization, and bacterial expression of a sesquiterpenoid biosynthetic gene (Ari1) from Penicillium roqueforti. J Biol Chem 268, 4543-4548.

Punt, P.J., Oliver, R.P., Dingemanse, M.A., Pouwels, P.H., and van den Hondel, C.A. (1987). Transformation of Aspergillus based on the hygromycin B resistance marker from Escherichia coli. Gene 56, 117-124.

Reyes-Dominguez, Y., Bok, J.W., Berger, H., Shwab, E.K., Basheer, A., Gallmetzer, A., et al. (2010). Heterochromatic marks are associated with the repression of secondary metabolism clusters in Aspergillus nidulans. Mol Microbiol 76, 1376-1386.

Sambrook, J., Fritsch, E., and Maniatis, T. (1989). Molecular Cloning: A Laboratory Mannual. Cold Spring Harbor Laboratory Press, New York), pp. 1-2222.

Sarikaya, B.O., Bayram, O., Valerius, O., Park, H.S., Irniger, S., Gerke, J., et al. (2010). LaeA control of velvet family regulatory proteins for light-dependent development and fungal cell-type specificity. PLoS Genet 6, e1001226.
Shaaban, M.I., Bok, J.W., Lauer, C., and Keller, N.P. (2010). Suppressor mutagenesis identifies a velvet complex remediator of Aspergillus nidulans secondary metabolism. Eukaryotic Cell 9, 1816-1824.

Shwab, E.K., and Keller, N.P. (2008). Regulation of secondary metabolite production in filamentous ascomycetes. Mycol Res 112, 225-230.

Snoek, I.S., van der Krogt, Z.A., Touw, H., Kerkman, R., Pronk, J., Bovenberg, R., et al. (2009). Construction of an hdfA Penicillium chrysogenum strain impaired in non-homologous endjoining and analysis of its potential for functional analysis studies. Fungal Genet Biol 46, 418-426.

Sprote, P., and Brakhage, A.A. (2007) The light-dependent regulator velvet $A$ of Aspergillus nidulans acts as a repressor of the penicillin biosynthesis. Arch Microbiol 188, 69-79.

Stinnett, S.M., Espeso, E.A., Cobeno, L., Araujo-Bazan, L., and Calvo, A.M. (2007). Aspergillus nidulans VeA subcellular localization is dependent on the importin alpha carrier and on light. Mol Microbiol 63, 242-255.

Tilburn, J., Scazzocchio, C., Taylor, G.G., Zabicky-Zissman, J.H., Lockington, R.A., and Davies, R.W. (1983). Transformation by integration in Aspergillus nidulans. Gene 26, 205-221.

Tusher, V.G., Tibshirani, R., and Chu, G. (2001). Significance analysis of microarrays applied to the ionizing radiation response. Proc Natl Acad Sci USA 98, 5116-5121.

van den Berg, M.A., Albang, R., Albermann, K., Badger, J.H., Daran, J.M., Driessen, A.J., et al. (2008). Genome sequencing and analysis of the filamentous fungus Penicillium chrysogenum. Nat Biotechnol 26, 1161-1168.

van den Berg, M.A., Bovenberg, R.A.L., Raamsdonk, L.M.L., Sutherland, J.D., deVroom, E., and Vollinga, R.C.R. Cephem compound. WO2004/106347. (2004).

van der Beek, C.P., and Roels, J.A. (1984). Penicillin production: biotechnology at its best. Antonie Van Leeuwenhoek 50, 625-639.

Verweij, P.E., Meis, J.F., van den Hurk, P., Zoll, J., Samson, R.A., and Melchers, W.J. (1995). Phylogenetic relationships of five species of Aspergillus and related taxa as deduced by comparison of sequences of small subunit ribosomal RNA. J Medi Vet Mycol 33, 185-190.

von Dohren, H. (2004). Biochemistry and general genetics of nonribosomal peptide synthetases in fungi. In Molecular Biotechnolgy of Fungal Beta-Lactam Antibiotics and Related Peptide Synthetases. A.A. Brakhage, ed. (Springer, Berlin), pp. 217-264. Wiemann, P., Brown, D.W., Kleigrewe, K., Bok, J.W., Keller, N.P., Humpf, H.U., et al. (2010). FfVel1 and FfLae1, components of a velvet-like complex in Fusarium fujikuroi, affect differentiation, secondary metabolism and virulence. Mol Microbiol 77, 972-994.

Address correspondence to: Daran Jean-Marc

Department of Biotechnology

Delft University of Technology Julianalaan 67

2628 BC Delft, The Netherlands

E-mail: j.g.daran@tudelft.nl 